\title{
Proteomic Biomarkers Panel: New Insights in Chronic Kidney Disease
}

\author{
Simona Mihai, ${ }^{1}$ Elena Codrici, ${ }^{1}$ Ionela Daniela Popescu, ${ }^{1}$ Ana-Maria Enciu, ${ }^{1,2}$ Elena Rusu, \\ Diana Zilisteanu, ${ }^{3,4}$ Radu Albulescu, ${ }^{1,5}$ Gabriela Anton, ${ }^{6}$ and Cristiana Tanase ${ }^{1,7}$ \\ ${ }^{1}$ Victor Babes National Institute of Pathology, Biochemistry-Proteomics Department, Splaiul Independentei 99-101, \\ Sector 5, 050096 Bucharest, Romania \\ ${ }^{2}$ Cellular and Molecular Medicine Department, Carol Davila University of Medicine and Pharmacy, \\ No. 8 B-dul Eroilor Sanitari, Sector 5, 050474 Bucharest, Romania \\ ${ }^{3}$ Fundeni Clinic of Nephrology, Carol Davila University of Medicine and Pharmacy, Soseaua Fundeni 258, \\ Sector 2, 022328 Bucharest, Romania \\ ${ }^{4}$ Fundeni Clinical Institute, Nephrology Department, Șoseaua Fundeni 258, Sector 2, 022328 Bucharest, Romania \\ ${ }^{5}$ National Institute for Chemical Pharmaceutical R\&D, Pharmaceutical Biotechnology Department, \\ Calea Vitan 112, Sector 3, 031299 Bucharest, Romania \\ ${ }^{6}$ Stefan S. Nicolau Institute of Virology, Molecular Virology Department, Soseaua Mihai Bravu 285, \\ Sector 3, 030304 Bucharest, Romania \\ ${ }^{7}$ Faculty of Medicine, Titu Maiorescu University, Strada Dâmbovnicului 22, Sector 4, 040441 Bucharest, Romania
}

Correspondence should be addressed to Cristiana Tanase; bioch@vbabes.ro

Received 7 April 2016; Accepted 27 July 2016

Academic Editor: Simone Ribero

Copyright ( $) 2016$ Simona Mihai et al. This is an open access article distributed under the Creative Commons Attribution License, which permits unrestricted use, distribution, and reproduction in any medium, provided the original work is properly cited.

Chronic kidney disease, despite being a "silent epidemic" disease, represents one of the main causes of mortality in general population, along with cardiovascular disease, which is the leading cause of poor prognosis for these patients. The specific objective of our study was to characterize the relationship between the inflammatory status, the bone disorders markers, and kidney failure in chronic kidney disease patient stages $2-4$, in order to design a novel biomarker panel that improves early disease diagnosis and therapeutic response, thus being further integrated into clinical applications. A panel of proteomic biomarkers, assessed by xMAP array, which includes mediators of inflammation (IL-6, TNF- $\alpha$ ) and mineral and bone disorder biomarkers (OPG, OPN, OCN, FGF-23, and Fetuin-A), was found to be more relevant than a single biomarker to detect early CKD stages. The association between inflammatory cytokines and bone disorders markers, IL-6, TNF- $\alpha$, OPN, OPG, and FGF-23, reflects the severity of vascular changes in $\mathrm{CKD}$ and predicts disease progression. Proteomic XMAP analyses shed light on a new approach to clinical evaluation for CKD staging and prognosis.

\section{Introduction}

Nowadays, chronic kidney disease (CKD) represents a worldwide major public burden and its prevalence continues to rise [1].

Over the past century, CKD, despite being a "silent epidemic" disease, represents one of the main causes of mortality in general population, alongside neoplasia, cardiovascular diseases, malnutrition, and infection, in the context of epidemiology landscape. Moreover, in Europe, CKD stages $1-5$ prevalence ranges from $3.3 \%$ to $17.3 \%[2,3]$.

Cardiovascular disease remains one of the leading causes of CKD poor prognosis, since early stages of CKD are associated with higher risk of subsequent coronary heart disease [4].

According to several clinical studies, $50 \%$ of patients with CKD die of cardiovascular causes, such as advanced calcific arterial and valvular disease; nonetheless, the processes of 
accelerated calcification in CKD remain poorly understood, and no therapies have been developed yet for disease prevention [5].

In CKD patients, screening for the presence of vascular calcification (VC) is suggested in current guidelines, since it is considered to be a cardiovascular risk marker and it is associated with a severalfold increase in morbidity and mortality risk, both in general population and in CKD, increasing steadily through the stages of CKD, peaking in CKD stage 5 patients.

Several proteins and factors are involved in passive and active processes that result in VC. In CKD population, various studies have identified circulating biomarkers that may be responsible for extraskeletal calcification and dysfunctions in mineral metabolism, which are features of CKD-mineral bone disorder (CKD-MBD) [6, 7].

Therefore, these observations have led to CKD-MBD study in association with cardiovascular diseases. These processes are interconnected and they have an important contribution to the morbidity and mortality rate of $\mathrm{CKD}$ patients $[8,9]$.

One of the main objectives in CKD therapy should be treating renal bone disease. The evaluation of CKD-MBD biochemical parameters (primarily phosphorus, calcium, parathyroid hormone, and vitamin D levels) as early as CKD stage 3, along with the assessment of bone status, should be considered in treatment decisions [10]. The prevalence of VC increases throughout the stages of $\mathrm{CKD}$ peaking in $\mathrm{CKD}$ stage 5 patients [7].

Cardiovascular calcification is an outstanding element of chronic inflammatory disorders associated with significant morbidity and mortality. Remarkably, CKD hastens atherosclerosis development and it has been demonstrated that CKD provokes excessive vascular inflammation and calcification [11].

Recent evidence also points towards alternative processes independent of osteogenic differentiation, including the release of matrix vesicles (e.g., secreted by macrophages) [5]. The understanding of the relationships between these mechanisms and signaling pathways could offer new mechanistic insight into the calcification process, and it may help lead to cardiovascular disease therapeutics in CKD patients $[5,11]$.

These data are also supported by genetic predisposition. Rutsch et al. [12] observed that $40-50 \%$ of coronary calcification cases can be attributed to genetics and several loci linked to coronary arterial calcification were identified [13, 14]. An implication of several single polymorphisms located at 9p21 locus near the cyclin genes has been suggested in the genesis of this pathology. These genes encode cyclins that may be broadly linked to cellular senescence and inflammation, though the accurate causative DNA sequences remain debatable $[14,15]$.

CKD is characterized by progressive loss of renal function, which results in reduced glomerular filtration rate (eGFR). Current clinical methods are accurate in diagnosing only advanced kidney dysfunction. In addition, there are no tools for predicting progression risk towards end-stage renal failure; thus, developing accurate biomarkers for prognosis of
CKD progression constitutes a clinical challenge. Therefore, efforts are directed towards earlier detection and better prognosis, in order to allow better therapeutic interventions to slow down or even prevent the progression of the disease in the future. Omics approaches, including proteomics, provide novel insights into disease mechanisms. They may improve CKD management, providing stage-specific biomarkers [1619].

In comparison with currently available markers, serum creatinine and urinary albumin, proteomic biomarkers may enable more accurate and earlier detection of renal pathology. Despite the "breaking point" being different in every patient, in some individuals serum creatinine levels remain normal despite loss of $>50 \%$ of renal function; consequently, additional biomarkers of renal function are needed. Biomarkers that would facilitate the noninvasive differential diagnosis of kidney diseases, detect early onset of kidney disease, monitor responses to therapy, and predict progression to hard end points, such as end-stage renal disease (ESRD) or death [7, $20,21]$, are needed, since they have potential for actual clinical implementation, which is an area to focus research on in the future [20, 22-25]. These biomarkers could prove very useful in terms of early detection and prognosis in CKD [7, 20, 2631].

Considering the above-mentioned aspects, the specific objective of this study was to characterize the relationship between the inflammatory status and the indicators of kidney failure and bone disorders, in order to design a novel biomarkers panel that might improve early disease diagnosis and therapeutic response, thus being further integrated into clinical practice.

\section{Materials and Methods}

\subsection{Patients and Samples}

2.1.1. Study Population. We prospectively included 86 patients (28\% female and $72 \%$ male; mean age 65 ) diagnosed with chronic kidney disease according to the KDIGO criteria, 20 with CKD stage 4 (35\% female and 65\% male; mean age 62), 52 with CKD stage 3 (33\% female and $67 \%$ male; mean age 66 ), and 14 with CKD stage 2 (23\% female and $77 \%$ male; mean age 65), hospitalized in Fundeni Clinic of Nephrology, Fundeni Clinical Institute, Bucharest, and 20 healthy controls. Before enrollment, written informed consent was obtained from all subjects, according to Helsinki Declaration and Ethics Committee that has approved the study. Patients with acute infection, known malignancy, acute heart failure, significant heart valvular disease, and chronic use of glucocorticoids and immunosuppressive agents were excluded.

2.1.2. Clinical and Laboratory Assessment. Clinical and anthropometric data were collected on the day of blood sampling: age, sex, weight, height, previous medical history, and concomitant treatment. Laboratory tests included hemoglobin, hematocrit, serum creatinine, urea, uric acid, glucose, total cholesterol, triglycerides, alkaline phosphatase, 
phosphate, calcium, albumin, and fibrinogen. Estimated glomerular filtrate rate (eGFR) was calculated using CKDEPI formula. Urinary protein excretion was measured from a $24 \mathrm{~h}$ urine sample. All blood samples were collected in the morning after an overnight fast and were stored at $-80^{\circ} \mathrm{C}$ until being analyzed.

2.2. xMAP Array and ELISA Analysis. The xMAP array was performed according to the manufacturers' protocols, and the plates were analyzed using Luminex 200 system. Cytokine levels and bone metabolism analytes were determined using the Milliplex MAP Human Bone Magnetic Bead Panel Kit from Merck-Millipore, Billerica, MA, USA, with 6 analytespecific bead sets (simultaneous quantification): proinflammatory cytokines IL- 6 and TNF- $\alpha$ and bone metabolism and disorder biomarkers: Osteoprotegerin (OPG), Osteocalcin (OCN), Osteopontin (OPN), and Fibroblast Growth Factor 23 (FGF-23). Briefly, the beads, which were provided within each kit, were incubated with buffer, cytokine standards (included in the kit), or samples in a 96-well plate at $4^{\circ} \mathrm{C}$ overnight. All further incubations with detection antibodies and Streptavidin Phycoerythrin Conjugate (SAPE) were performed at room temperature in the dark with shaking at $800 \mathrm{rpm}$. Multiplex data acquisition and analysis were performed using xPONENT 3.1 software; the calibration curves were generated with a 5-parameter logistic fit.

Fetuin-A serum levels were assessed using Quantikine ${ }^{\circledR}$ ELISA Human Fetuin A Immunoassay kit, R\&D Systems, Inc., USA, according to the manufacturer's instructions.

Duplicate samples were used for all specimens and the average concentrations were used for statistical analysis.

2.3. Statistical Analysis. Differences between CKD sample group and control were analyzed using Student's $t$-test. A twotailed $p<0.05$ was considered statistically significant. The chi-square test $\left(\chi^{2} ; P\right)$ was used to determine the significance of the association between inflammatory cytokines, bone metabolism, and disorder biomarkers in CKD and control groups. Pearson correlation $(r)$ was used to explore the association between different biomarkers expression, together with clinical parameters. The threshold values for the analyses were established in accordance with the mean values of the studied groups. Statistical analysis was performed using SPSS 19.0 software. Graphs were realized with GraphPad Prism software (GraphPad Software Inc., La Jolla, CA).

\section{Results and Discussion}

Given the fact that a large number of cytokines orchestrate the inflammatory response, the extent to which inflammation plays a role in increasing the risk of bone/mineral disorders in CKD remains unclear. Progressive renal failure in CKD contributes to abnormalities in mineral/bone metabolismcalcium, phosphorous, PTH, Vitamin D, and vascular calcifications [32].

3.1. Inflammation and $C K D$. Inflammation represents a hallmark of $\mathrm{CKD}$ and the degree to which inflammation is
TABLE 1: Correlation between inflammatory cytokines and CKD biomarkers in stage 4 (Pearson correlation).

\begin{tabular}{lccccccc}
\hline & IL-6 & TNF- $\alpha$ & OPG & OCN & OPN & FGF-23 & Fetuin-A \\
\hline IL-6 & 1 & & & & & & \\
TNF- $\alpha$ & 0.64 & 1 & & & & & \\
OPG & -0.01 & 0.28 & 1 & & & & \\
OCN & 0.67 & 0.69 & 0.21 & 1 & & & \\
OPN & 0.59 & 0.72 & 0.04 & 0.35 & 1 & & \\
FGF-23 & 0.24 & -0.21 & -0.36 & 0.06 & 0.07 & 1 & \\
Fetuin-A & -0.37 & -0.62 & -0.31 & -0.39 & -0.67 & 0.23 & 1 \\
\hline
\end{tabular}

TABLE 2: Correlation between inflammatory cytokines and CKD biomarkers in stage 3 (Pearson correlation).

\begin{tabular}{lccccccc}
\hline & IL-6 & TNF- $\alpha$ & OPG & OCN & OPN & FGF-23 & Fetuin-A \\
\hline IL-6 & 1 & & & & & & \\
TNF- $\alpha$ & 0.147 & 1 & & & & & \\
OPG & 0.144 & 0.132 & 1 & & & & \\
OCN & 0.240 & -0.079 & 0.063 & 1 & & & \\
OPN & 0.173 & 0.106 & 0.53 & 0.083 & 1 & & \\
FGF-23 & 0.152 & 0.072 & 0.159 & 0.134 & 0.23 & 1 & \\
Fetuin-A & 0.196 & -0.03 & -0.048 & 0.072 & 0.015 & -0.0006 & 1 \\
\hline
\end{tabular}

related to loss in kidney function, eGFR, remains an open question. Some studies revealed increased circulating levels of proinflammatory cytokines IL- 6 and TNF- $\alpha$ in patients with kidney dysfunction [33]. Moreover, inflammation status in CKD seems to be correlated with CKD evolution and complications, like cardiovascular disease [33, 34].

Okada et al. study supported the assumption that IL6 genetic variations may lead to CKD and the assessment of the genotypes involved could identify the risk of CKD development [35]. Barreto et al. also showed that IL-6 levels tend to rise as CKD progressed, with the increase becoming statistically significant in CKD stages 4 and 3 [36].

According to our data in this study, the mediators of the inflammatory response IL- 6 and TNF- $\alpha$ have been overexpressed in all CKD groups ( $t$-test; $p<0.001$; Figures 1(a) and $1(\mathrm{c}))$. Statistical analysis ( $t$-test) shows that IL-6 level was highest in CKD stage 4 ( $p<0.001$ ), being 11-fold higher than control, while, for CKD stages 3 and 2, the expression was also increased, 6 -fold $(p<0.001)$ and 2 -fold $(p=0.005)$, respectively, by comparison with control group (Figures 1(b) and 2).

In CKD stage 4, IL-6 enhanced expression was directly correlated with TNF- $\alpha(r=0.64)$, OCN $(r=0.67)$, and OPN levels ( $r=0.59$ ) (Pearson correlation). In stage 2 of CKD, we have noticed a positive correlation between IL- 6 and TNF- $\alpha$ $(r=0.58)$, OPG $(r=0.63)$, and OPN $(r=0.52)$, and, on the other hand, a negative correlation with Fetuin-A $(r=-0.5)$ (Pearson correlation), as it is depicted in Tables 1,2, and 3.

TNF- $\alpha$ displays an increased level in serum of more than 3.6-fold $(p<0.001)$ in CKD stage 4 , recording a decrease with disease stage decrease as well, as follows: 2.8 -fold ( $p=$ $0.006)$ in CKD stage 3 and 1.7-fold in CKD stage $2(p=0.01)$; 


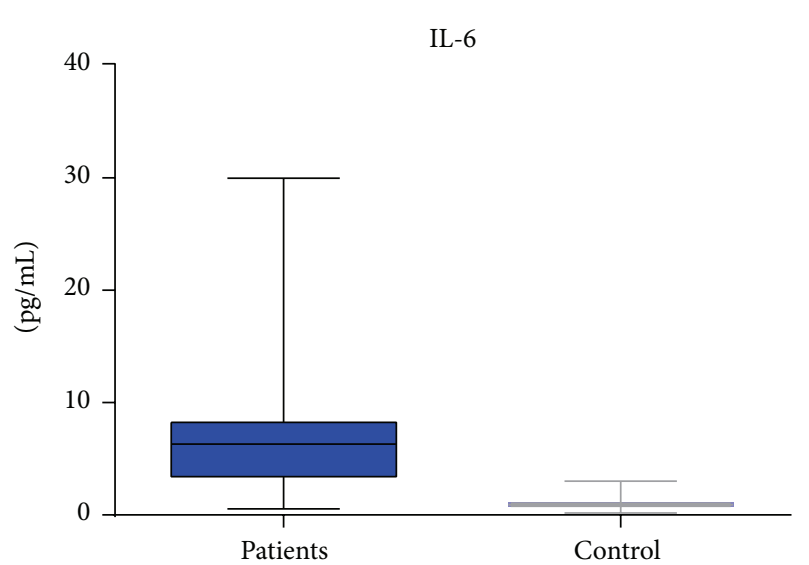

(a)

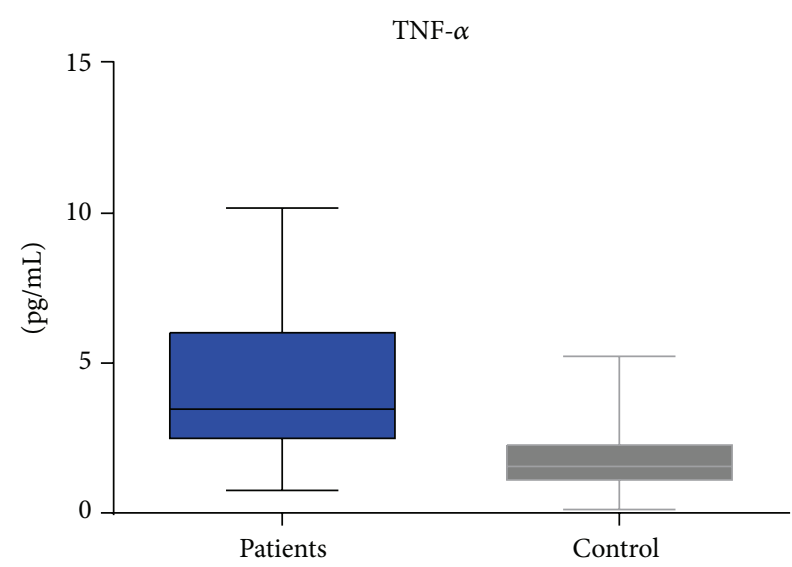

(c)

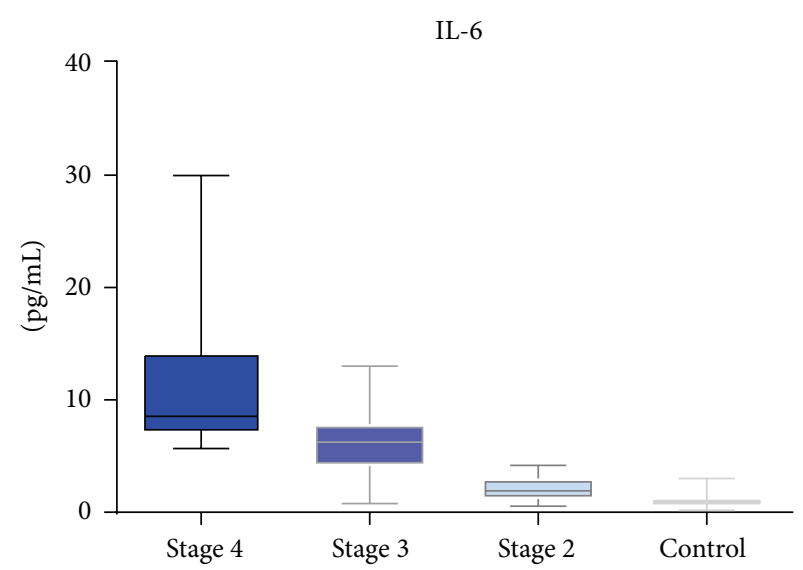

(b)

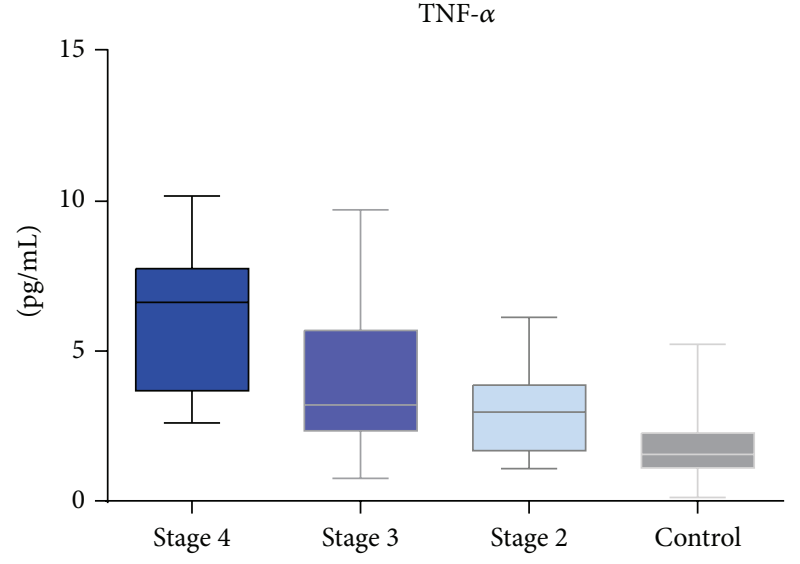

(d)

FIGURE 1: Serum levels of proinflammatory cytokines IL-6 and TNF- $\alpha$, in CKD patients versus control, assessed by xMAP array.

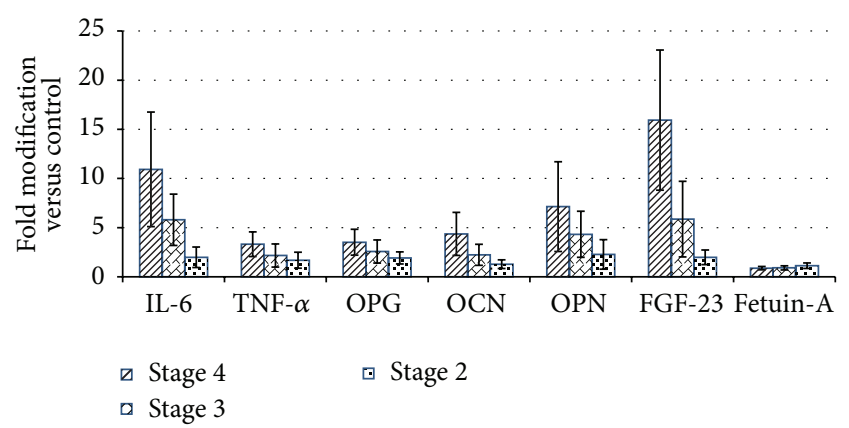

FIGURE 2: Modulation of serum biomarkers level in CKD stages. The data represent group averages of fold modification versus controls with standard deviations.

these results are also related to renal failure (eGFR). Details on expression of TNF- $\alpha$ are provided in Figures 1(d) and 2.

In CKD stage 4 , TNF- $\alpha$ enhanced expression is positively correlated with OCN $(r=0.69)$ and with OPN $(r=0.72)$ and negatively correlated with Fetuin-A $(r=-0.62)$. In CKD
TABLE 3: Correlations between inflammatory cytokines and CKD biomarkers in stage 2 (Pearson correlation).

\begin{tabular}{lccccccc}
\hline & IL-6 & TNF- $\alpha$ & OPG & OCN & OPN & FGF-23 & Fetuin-A \\
\hline IL-6 & 1 & & & & & & \\
TNF- $\alpha$ & 0.583 & 1 & & & & & \\
OPG & 0.638 & 0.375 & 1 & & & & \\
OCN & 0.003 & 0.294 & 0.054 & 1 & & & \\
OPN & 0.525 & 0.511 & 0.011 & 0.286 & 1 & & \\
FGF-23 & -0.125 & 0.136 & -0.123 & -0.2 & 0.334 & 1 & \\
Fetuin-A & -0.503 & -0.361 & -0.655 & -0.275 & 0.158 & 0.28 & 1 \\
\hline
\end{tabular}

stage 2 , TNF- $\alpha$ was positively correlated with OPN $(r=0.51)$, as it is shown in Tables 1,2, and 3 .

It has been shown that IL-6, a "bad" cytokine that could promote atherosclerosis [37], might be more helpful than TNF- $\alpha$ in CKD patients classification on stages [38].

Spoto et al. also concluded that inflammation is related to renal failure, with high IL-6 levels seen in CKD early stages exclusively; however, their data showed a negative correlation 
between TNF- $\alpha$ levels and eGFR, indicating differences in the dynamics of the relationship between the above-mentioned cytokines and renal function [39]. Our data is in accordance with the increase of IL- 6 in CKD, but we found a positive correlation between TNF- $\alpha$ and renal function.

The increased serum levels of IL- 6 and TNF- $\alpha$ in CKD are in accordance with other studies, which mention that proinflammatory cytokines increase is linked to disease progression [40].

\subsection{Markers of Mineral and Bone Disorders in CKD}

3.2.1. Osteoprotegerin (OPG). OPG is considered a member of the TNF receptor family, considered to be correlated with the vascular dysfunction and further with cardiovascular disease, the common problem encountered in patients with CKD. Yilmaz et al. were among the first ones to mention the potential role of OPG in CKD patients stratification for cardiovascular risk, along with eGFR and FGF-23, in a CKD group not undergoing dialysis [41].

In this regard, according to another study, OPG increased expression could be linked to medial calcifications in aorta and renal arteries; thus its expression is recognized as a protective mechanism against vascular calcifications [6]. Thereby, the RANK/RANKL/OPG signaling pathway was found to be closely related to atherosclerosis progression [42].

Our data suggest a statistically significant increased expression of OPG in CKD patients group compared with control $(p<0.001$, Figure 3(a)). The same results were found in a study by Demir et al. [43]. The upward trend of OPG levels is also maintained with regard to the distribution on CKD stages, as follows: 3.5-fold higher in CKD stage 4, 2.5fold higher in CKD stage 3, and 2-fold higher in CKD stage 2 $(p<0.001$ for all stages, Figures 3(b) and 2).

Despite the fact that several studies propose a direct involvement action of IL-6 in the increasing level of expression of OPG, we can conclude, based on our data, that the level of OPG in serum correlates with the expression level of IL-6 in patients of CKD stage 2 only $(r=0.63)$. Given the relatively small number of patients included in this study, further research is necessary to fully understand the therapeutic and biomarker potential of OPG in patients with kidney disease.

Morena et al. were among the first that also mentioned that increased OPG levels were correlated with the progression of coronary artery calcification (CAC) in a CKD nondialyzed group [44].

It was observed that OPG knockout mice develop severe calcifications, thus the potential protective role of OPG against vascular calcification being proposed [45].

Furthermore, Lewis et al. concluded that OPG might be a crucial biomarker in CKD stages 3-5 patients with poor longterm prognosis, based on their results showing that OPG high levels were correlated with the progress in renal dysfunction [46].

3.2.2. Osteocalcin (OCN). Considering the osteoblastic activity of OCN, this marker might be directly involved in bonevascular axis [47, 48] and its systemic and local effects could be potentially related to bone remodeling, vascular calcification, and energy metabolism [49].

We found that OCN circulating levels were increased 4.6-fold in CKD stage $4(p<0.01)$ and 2 -fold $(p<0.01)$ and 1.3 -fold ( $p=0.05$ ) in CKD stages 3 and 2, respectively, thus revealing an overexpression of OCN in CKD patients versus control ( $p<0.001)$ (Figures 3(c), 3(d), and 2). The OCN serum levels, in association with inflammatory markers IL6 and TNF- $\alpha$, showed significant correlation with regard to CKD stage 4 only ( $r=0.67$ and $r=0.69$ ) (Tables 1,2 , and 3 ).

Other studies observed that OCN was inversely correlated to age and IL-6, in CKD hemodialysis patients [50].

Since the controversy still exists, further research and large clinical trials are needed to clearly explain the connections between the immune system and bone-vascular axis.

3.2.3. Osteopontin $(O P N)$. In $\mathrm{CKD}$ patients, starting with early stages, Barreto et al. have noticed increased OPN levels compared with control and have also related a positive correlation of OPN with the inflammatory markers $[4,51]$.

In a univariate linear regression assessment, OPN was found to be directly correlated with inflammation markers like IL-6, C-reactive protein (CRP), and intact parathyroid hormone (iPTH), concluding that OPN could play an important role in the pathway where inflammation enhanced CKD poor prognosis [51].

Lorenzen et al. also found a possible link between OPN and inflammation markers (IL-6, CRP) in hypertensive patients [52].

Our results were in agreement with the above-mentioned studies and revealed significant differences between the control group and patients with CKD $(p<0.001)$, being increased more than 2 -fold in CKD stage $2(p=0.01)$, rising at 4 -fold in CKD stage $3(p<0.001)$ and 7 -fold in CKD stage $4(p<0.0001)$ (Figures 3(e), 3(f), and 2).

The threshold values for the analyses were established in accordance with the mean values of the studied groups. A negative correlation was observed between OPN and FetuinA serum levels of CKD stage 4 patients $(r=-0.67)$, and a positive correlation was found between OPN and IL-6 ( $r=$ $0.59)$ and TNF- $\alpha(r=0.72)$. In CKD stage 3 , a statistical correlation between OPN and OPG was observed $(r=0.53)$. A statistical correlation was also found with IL-6 $(r=0.52)$ and TNF- $\alpha(r=0.51)$ in CKD stage 2 (Tables 1,2 , and 3$)$.

3.2.4. Fibroblast Growth Factor 23 (FGF-23). FGF-23 is a phosphaturic hormone with elevated levels in early CKD stages, before mineral and bone disorders become obvious [53], and might be associated with endothelial dysfunction [54] and greater risk of congestive heart failure (CHF) and atherosclerotic events in patients with CKD stages 2-4 [55].

Nonetheless, in another study, FGF23 appears not to be an early marker of CKD, in elderly patients (age over 65) [56].

It is generally considered that CKD plays the most important role in increasing FGF-23 levels; in this view, FGF-23 high levels appeared to be independently linked to CKD prognosis $[57,58]$, although the mechanisms are poorly understood [59]. 


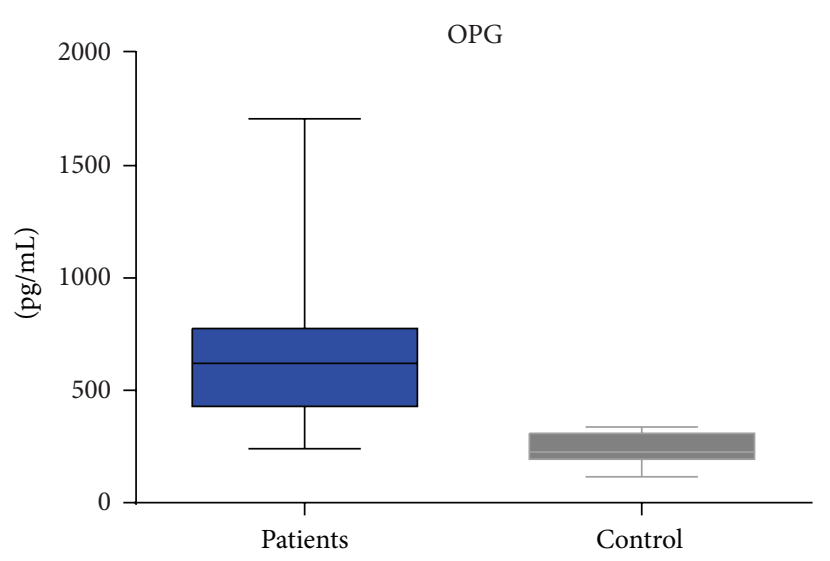

(a)

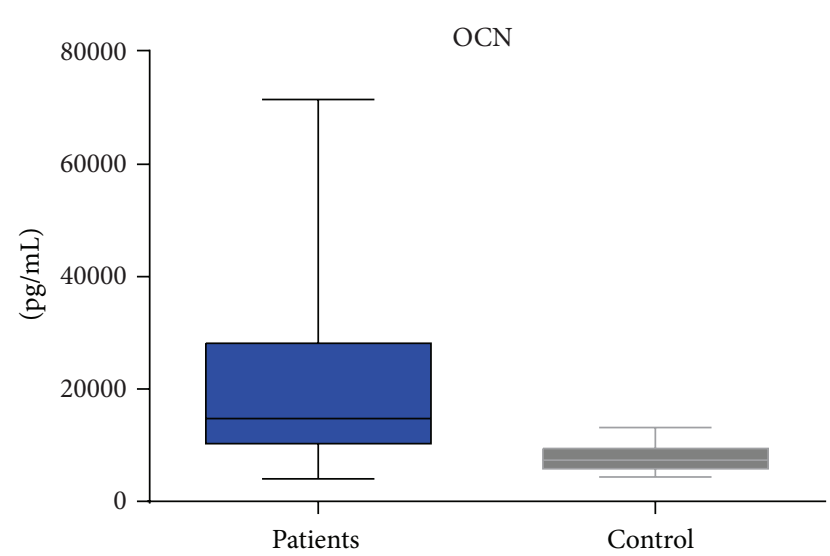

(c)

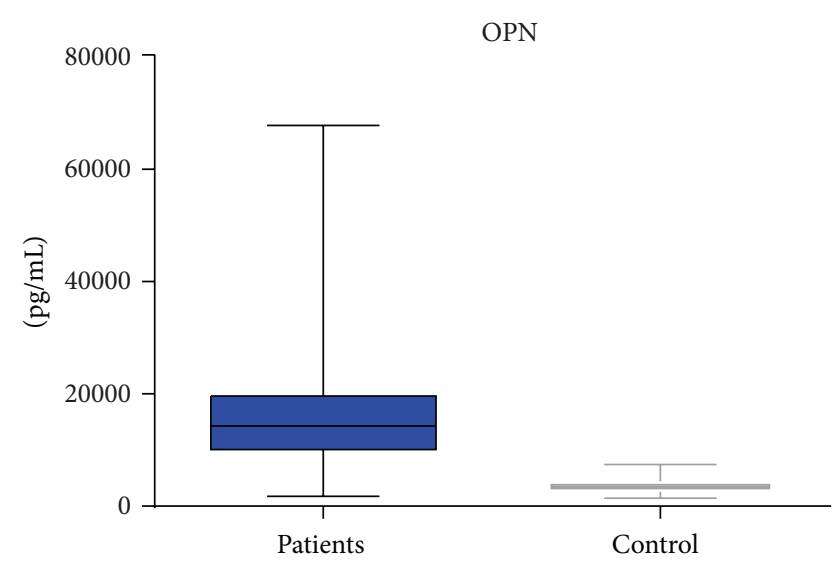

(e)

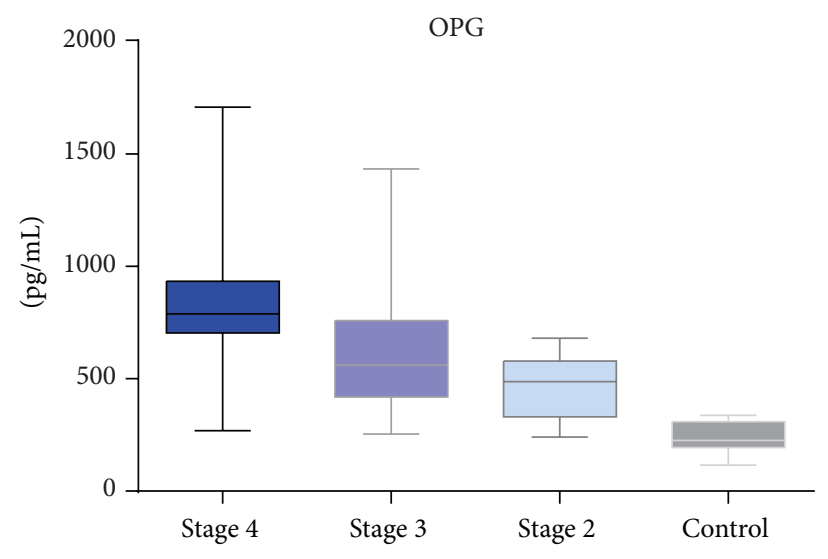

(b)

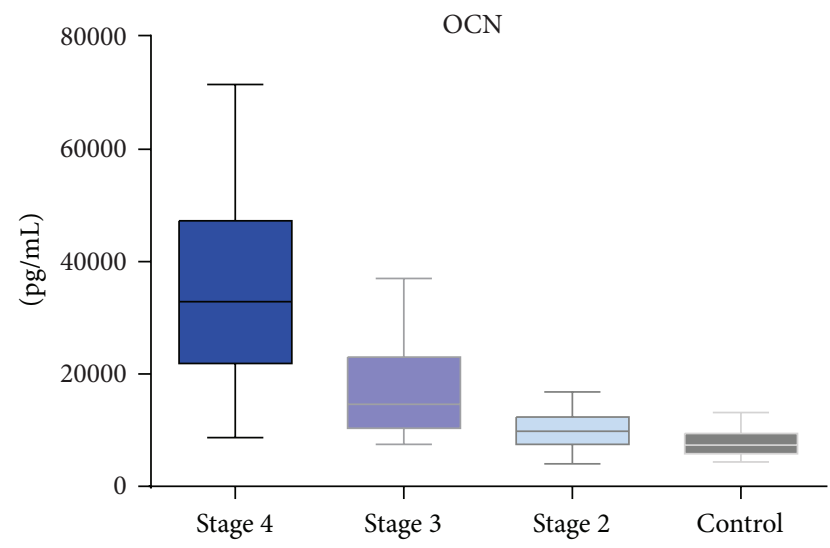

(d)

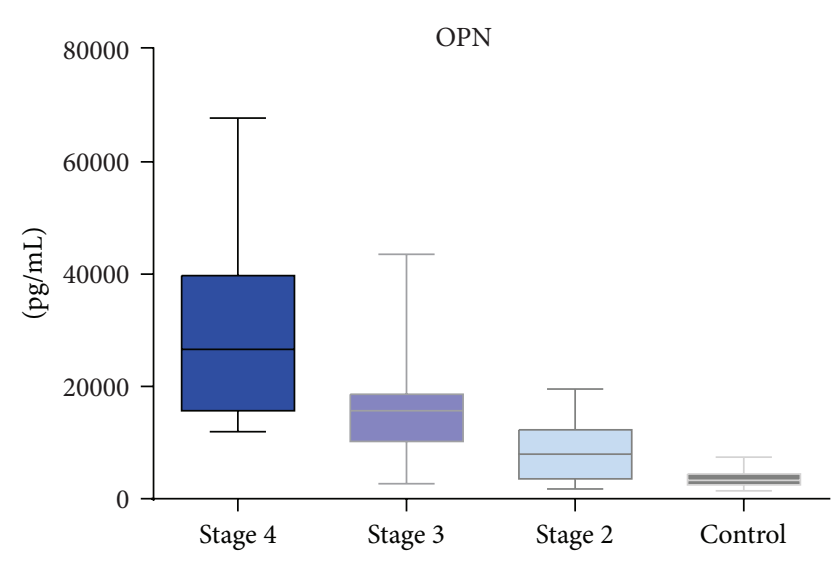

(f)

FIGURE 3: Serum level of OPG, OCN, and OPN in CKD patients compared with control, by xMAP array.

Desjardins et al. suggest that plasma FGF-23 could be considered an independent biomarker of vascular calcification in patients with CKD, starting from early stages [60].

We have found that FGF-23 levels were significantly enhanced in CKD patients $(p<0.001)$ (Figure 4(a)). Serum levels of FGF-23 showed a gradual increase, reaching the highest levels in patients with CKD stage $4(p<0.0001)$, being 16-fold higher than in the control group. According to previously analyzed biomarkers, serum FGF-23 levels still showed a significant increase of 6 -fold $(p<0.001)$ in CKD stage 3 and 2 -fold $(p<0.001)$ for CKD stage 2 (Figures 4(b) and 2). Although FGF-23 has been identified to be significantly overexpressed in CKD stages 2-4, there were no statistical correlations with the other multiplexed analyzed biomarkers (according to Pearson correlations).

3.2.5. Fetuin-A. Among the multiple players involved in vascular calcification pathogenesis, Fetuin-A is considered to 
FGF-23

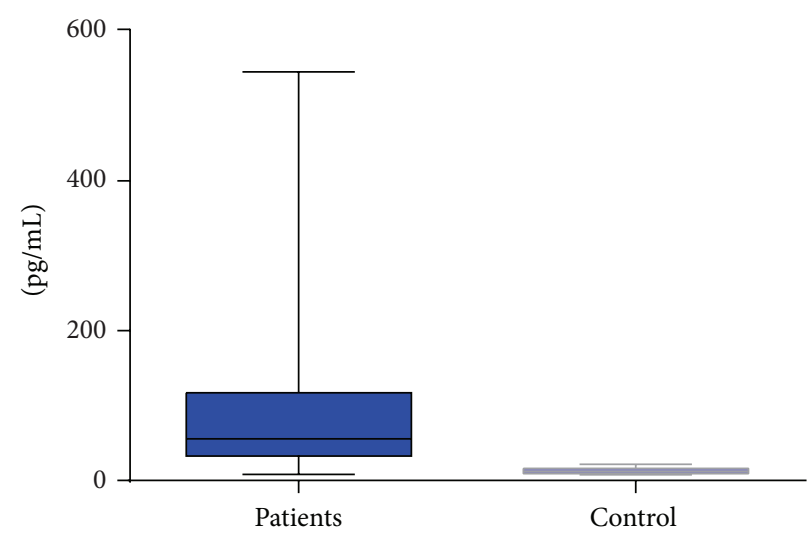

(a)

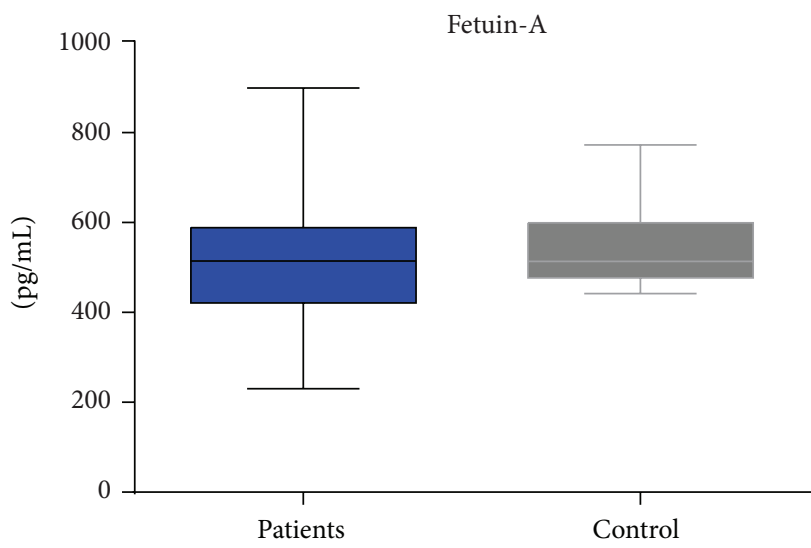

(c)

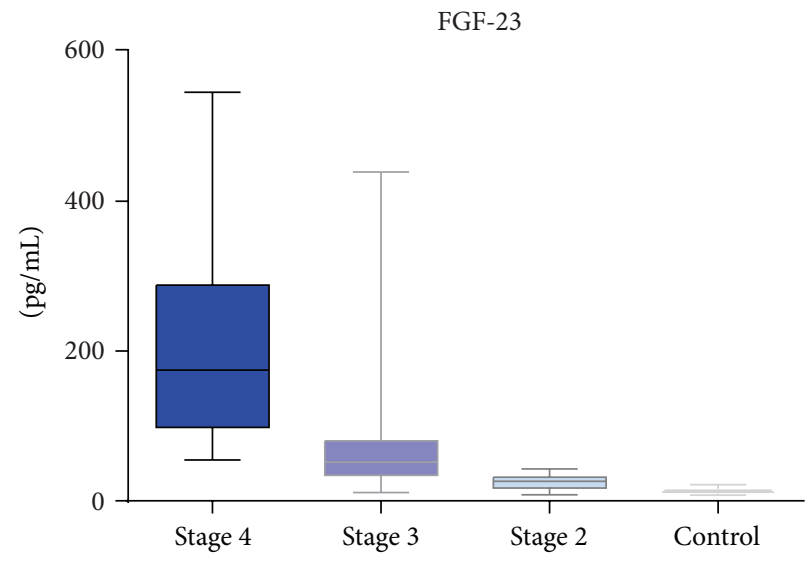

(b)

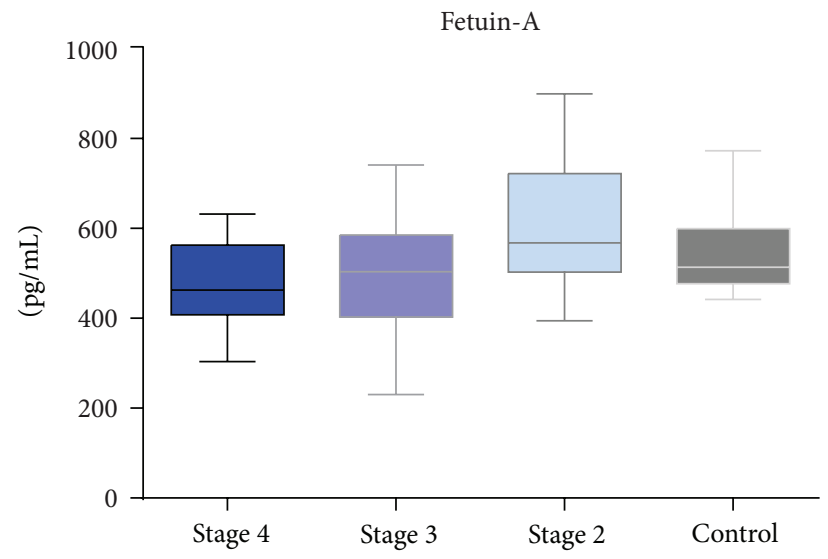

(d)

Figure 4: Serum level of FGF-23 and Fetuin-A in CKD patients compared with control, assessed by xMAP array.

be an inhibitory molecule; thus CKD patients are assumed to experience a Fetuin-A deficiency, which might be considered a common feature of this disease [61].

We have noticed that the level of Fetuin-A in CKD patients was decreased compared with control $(p=0.15)$. The highest decrease was found in CKD stage 4 , being of 0.85 fold $(p=0.02)$, followed by stage 3 with 0.91 -fold $(p=0.05)$ and stage 2 with 1.13-fold ( $p=0.13$ ) ( $t$-test) (Figures 4(c), $4(\mathrm{~d})$, and 2). Fetuin-A presented a negative correlation with TNF- $\alpha(r=-0.61)$ and OPN $(r=-0.67)$ in CKD stage 4 , while in CKD stage 2 Fetuin-A was negatively correlated with IL-6 $(r=-0.5)$ and OPG $(r=-0.6)$ (Tables 1,2 , and 3$)$.

Smith et al. also reported an association between FetuinA decreased levels and inflammatory markers, also with procalcific cytokine, explaining the potential involvement of this biomarker in coronary calcification and aortic stiffness [62].

3.3. Correlations between CKD Markers and Inflammatory Status. We have observed a strong correlation between IL6 and eGFR $\left(\chi^{2}=16.8 ; P<0.01\right)$, TNF- $\alpha\left(\chi^{2}=7.9\right.$; $P<0.005)$, OPN ( $\left.\chi^{2}=5.4 ; P<0.02\right)$, OPG $\left(\chi^{2}=8.28\right.$; $P=0.04)$, and FGF-23 ( $\left.\chi^{2}=5 ; P=0.02\right)$. TNF- $\alpha$ was correlated with FGF-23 $\left(\chi^{2}=7.4 ; P=0.006\right)$ and Fetuin-A $\left(\chi^{2}=5.9 ; P=0.001\right)$. Strong correlations were also found between eGFR and OCN $\left(\chi^{2}=6.2 ; P=0.01\right)$ and FGF$23\left(\chi^{2}=19.9 ; P<0.001\right)$; also OCN correlated with OPN $\left(\chi^{2}=5.3 ; P=0.02\right)$ and FGF-23 $\left(\chi^{2}=6.9 ; P=0.008\right)$ in all CKD groups. The above-mentioned correlations, chi-square test $\left(\chi^{2} ; P\right)$, between analyzed inflammatory mediators and mineral/bone disorders markers, alongside with eGFR, are shown in Table 4.

According to our results, we conclude that a crosstalk between bone, vasculature, and renal function exists in CKD, representing a major risk factor for cardiovascular morbidity and mortality.

In CKD early stage 2, an increased expression was observed for 6 out of the 7 analyzed biomarkers. From our data, circulating levels of IL- 6 , TNF- $\alpha$, OPG, OCN, OPN, and FGF-23 were statistically increased $(P<0.05)$ in CKD stage 2 , while Fetuin-A showed a slight alteration over control, but with no statistical significance $(P=0.13)$.

At a first glance, proteomic biomarkers offer the hope of improving the management of patients with CKD starting with early stages, yet more studies are needed to establish the diagnostic and prognostic value of these biomarkers. 


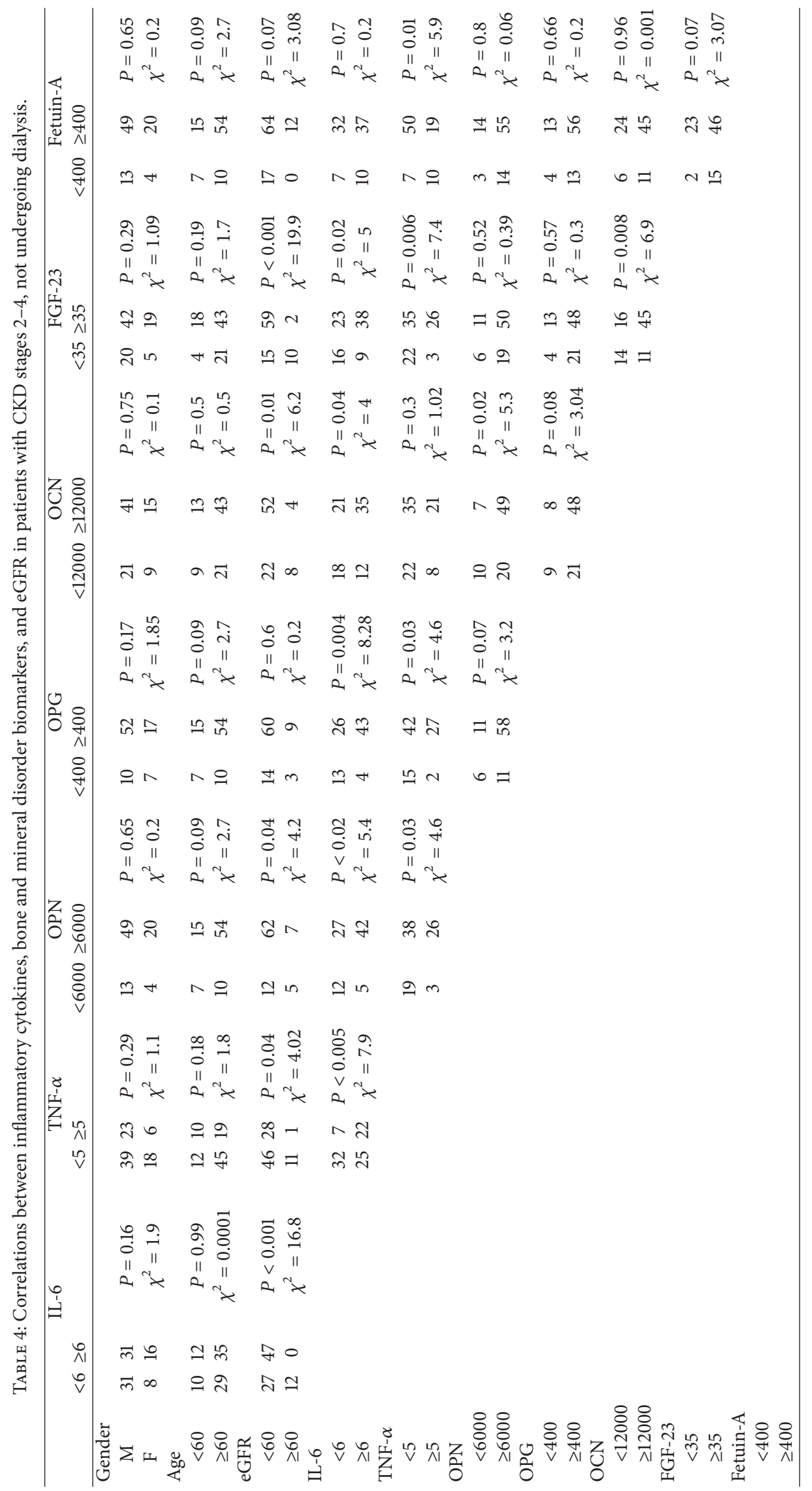




\section{Conclusions}

The present study highlights the potential clinical utility of a multiplexed biomarker panel in CKD. Out of all analyzed candidate biomarkers, a panel which includes mediators of inflammation (IL-6, TNF- $\alpha$ ) and mineral and bone disorder biomarkers (OPG, OPN, OCN, FGF-23, and Fetuin-A) was found to be more relevant than a single biomarker to detect patients in early CKD stages. We have noticed a positive correlation between the biomarkers panel of IL-6, OCN, and FGF-23 and renal failure progression (eGFR) in all CKD groups. The association between inflammatory cytokines and bone disorders markers, OPN, OPG, and FGF-23, reflects the severity of the vascular changes in $\mathrm{CKD}$ and predicts the disease progression. Proteomic xMAP analyses shed light on clinical evaluation for CKD staging and prognosis. Thus, new evidence has emerged within the relationship between bone and vascular pathology, especially in CKD patients, encouraging further investigations in the area.

\section{Competing Interests}

The authors declare that they have no competing interests.

\section{Authors' Contributions}

The authors contributed equally to this work.

\section{Acknowledgments}

This work was partly supported by a grant of the Romanian National Authority for Scientific Research and Innovation PNII 93/2012 (PN-II-PT-PCCA-2011-3.2-0794), PNII 265/2014, PNII 192/2014, and PN 16.22.04.01. The authors would like to thank Irina Radu, certified translator in Medicine-Pharmacy, certificate credentials: series: E no. 0048, for professional linguistic assistance.

\section{References}

[1] A. Gluba-Brzózka, M. Michalska-Kasiczak, B. Franczyk et al., "Markers of increased atherosclerotic risk in patients with chronic kidney disease: a preliminary study," Lipids in Health and Disease, vol. 15, no. 1, article 32, 2016.

[2] C. Zoccali, A. Kramer, and K. J. Jager, "Epidemiology of CKD in Europe: an uncertain scenario," Nephrology Dialysis Transplantation, vol. 25, no. 6, pp. 1731-1733, 2010.

[3] K. Bruck, V. S. Stel, G. Gambaro et al., "CKD prevalence varies across the european general population," Journal of the American Society of Nephrology, vol. 27, no. 7, pp. 2135-2147, 2016.

[4] A. Gluba-Brzozka, M. Michalska-Kasiczak, B. Franczyk et al., "Markers of increased atherosclerotic risk in patients with chronic kidney disease: a preliminary study," Lipids in Health and Disease, vol. 15, no. 1, p. 22, 2016.

[5] E. Aikawa, M. Aikawa, P. Libby et al., "Arterial and aortic valve calcification abolished by elastolytic cathepsin S deficiency in chronic renal disease," Circulation, vol. 119, no. 13, pp. 1785-1794, 2009.
[6] M. L. Ford, E. R. Smith, L. A. Tomlinson, P. K. Chatterjee, C. Rajkumar, and S. G. Holt, "FGF-23 and osteoprotegerin are independently associated with myocardial damage in chronic kidney disease stages 3 and 4 . Another link between chronic kidney disease-mineral bone disorder and the heart," Nephrology Dialysis Transplantation, vol. 27, no. 2, pp. 727-733, 2012.

[7] S. Liabeuf, H. Okazaki, L. Desjardins et al., "Vascular calcification in chronic kidney disease: are biomarkers useful for probing the pathobiology and the health risks of this process in the clinical scenario?" Nephrology Dialysis Transplantation, vol. 29, no. 7, pp. 1275-1284, 2014.

[8] Kidney Disease: Improving Global Outcomes (KDIGO) CKDMBD Work Group, "KDIGO clinical practice guideline for the diagnosis, evaluation, prevention, and treatment of chronic kidney disease-mineral and bone disorder (CKD-MBD)," Kidney International, vol. 76, supplement 113, pp. S1-S130, 2009.

[9] D. Brancaccio, M. Gallieni, S. Pasho et al., "Pathogenesis and treatment of vascular calcification in CKD," Giornale Italiano di Nefrologia, vol. 26, supplement 45, pp. S20-S27, 2009.

[10] G. London, D. Coyne, K. Hruska, H. H. Malluche, and K. J. Martin, "The new kidney disease: Improving global outcomes (KDIGO) guidelines-expert clinical focus on bone and vascular calcification," Clinical Nephrology, vol. 74, no. 6, pp. 423-432, 2010.

[11] M. Rogers, C. Goettsch, and E. Aikawa, "Medial and intimal calcification in chronic kidney disease: stressing the contributions," Journal of the American Heart Association, vol. 2, no. 5, article e000481, 2013.

[12] F. Rutsch, Y. Nitschke, and R. Terkeltaub, "Genetics in arterial calcification: pieces of a puzzle and cogs in a wheel," Circulation Research, vol. 109, no. 5, pp. 578-592, 2011.

[13] M. A. Hofmann Bowman and E. M. McNally, "Genetic pathways of vascular calcification," Trends in Cardiovascular Medicine, vol. 22, no. 4, pp. 93-98, 2012.

[14] C. J. O'donnell, M. Kavousi, A. V. Smith et al., "Genome-wide association study for coronary artery calcification with followup in myocardial infarction," Circulation, vol. 124, no. 25, pp. 2855-2864, 2011.

[15] S. Evrard, P. Delanaye, S. Kamel et al., "Vascular calcification: from pathophysiology to biomarkers," Clinica Chimica Acta, vol. 438, pp. 401-414, 2015.

[16] D. Philippe Rouet, E. Nkuipou-Kenfack, F. Duranton et al., "Assessment of metabolomic and proteomic biomarkers in detection and prognosis of progression of renal function in chronic kidney disease," PLoS ONE, vol. 9, no. 5, Article ID e96955, 2014.

[17] M. Breit and K. M. Weinberger, "Metabolic biomarkers for chronic kidney disease," Archives of Biochemistry and Biophysics, vol. 589, pp. 62-80, 2016.

[18] M. Neagu, "The immune system-a hidden treasure for biomarker discovery in cutaneous melanoma," Advances in Clinical Chemistry, vol. 58, pp. 89-140, 2012.

[19] C. P. Tanase, M. Neagu, and R. Albulescu, "Key signaling molecules in pituitary tumors," Expert Review of Molecular Diagnostics, vol. 9, no. 8, pp. 859-877, 2009.

[20] H. Mischak, C. Delles, A. Vlahou, and R. Vanholder, "Proteomic biomarkers in kidney disease: issues in development and implementation," Nature Reviews Nephrology, vol. 11, no. 4, pp. 221232, 2015.

[21] I. D. Popescu, E. Codrici, L. Albulescu et al., "Potential serum biomarkers for glioblastoma diagnostic assessed by proteomic approaches," Proteome Science, vol. 12, no. 1, article 47, 2014. 
[22] M. J. Pena, D. De Zeeuw, H. Mischak et al., "Prognostic clinical and molecular biomarkers of renal disease in type 2 diabetes," Nephrology Dialysis Transplantation, vol. 30, supplement 4, pp. iv86-iv95, 2015.

[23] A. Bulman, M. Neagu, and C. Constantin, "Immunomics in skin cancer-improvement in diagnosis, prognosis and therapy monitoring," Current Proteomics, vol. 10, no. 3, pp. 202-217, 2013.

[24] M. Neagu, C. Constantin, and C. Tanase, "Immune-related biomarkers for diagnosis/prognosis and therapy monitoring of cutaneous melanoma," Expert Review of Molecular Diagnostics, vol. 10, no. 7, pp. 897-919, 2010.

[25] C. P. Tanase, R. Albulescu, and M. Neagu, "Application of 3D hydrogel microarrays in molecular diagnostics: advantages and limitations," Expert Review of Molecular Diagnostics, vol. 11, no. 5, pp. 461-462, 2011.

[26] C. P. Tanase, M. Neagu, R. Albulescu, E. Codorean, and S. O. Dima, "Biomarkers in the diagnosis and early detection of pancreatic cancer," Expert Opinion on Medical Diagnostics, vol. 3, no. 5, pp. 533-546, 2009.

[27] M. Neagu, C. Constantin, G. Manda, and I. Margaritescu, "Biomarkers of metastatic melanoma," Biomarkers in Medicine, vol. 3, no. 1, pp. 71-89, 2009.

[28] M. Neagu, C. Constantin, and S. Zurac, "Immune parameters in the prognosis and therapy monitoring of cutaneous melanoma patients: experience, role, and limitations," BioMed Research International, vol. 2013, Article ID 107940, 13 pages, 2013.

[29] C. Pistol-Tanase, E. Raducan, S. O. Dima et al., "Assessment of soluble angiogenic markers in pancreatic cancer," Biomarkers in Medicine, vol. 2, no. 5, pp. 447-455, 2008.

[30] C. P. Tanase, A.-M. Enciu, S. Mihai, A. I. Neagu, B. Calenic, and M. L. Cruceru, "Anti-cancer therapies in high grade gliomas," Current Proteomics, vol. 10, no. 3, pp. 246-260, 2013.

[31] I. D. Popescu, R. Albulescu, E. Raducan, A. Dinischiotu, and C. Tănase, "Applications of SELDI-TOF technology in cancer biomarkers discovery," Romanian Biotechnological Letters, vol. 15, no. 5, pp. 5654-5667, 2010.

[32] F. M. Shaheen, R. Kurpad, A. Al-Sayyari et al., "Multinational observational study on clinical practices and therapeutic management of mineral and bone disorders in patients with chronic kidney disease stages 4, 5, and 5D: the OCEANOS study," Saudi Journal of Kidney Diseases and Transplantation, vol. 27, no. 2, article 290, 2016.

[33] A. G. Abraham, A. Darilay, H. McKay et al., "Kidney dysfunction and markers of inflammation in the multicenter AIDS cohort study," The Journal of Infectious Diseases, vol. 212, no. 7, pp. 1100-1110, 2015.

[34] L. Hénaut, M. D. Sanchez-Nino, G. Aldamiz-Echevarría Castillo, A. B. Sanz, and A. Ortiz, “Targeting local vascular and systemic consequences of inflammation on vascular and cardiac valve calcification," Expert Opinion on Therapeutic Targets, vol. 20, no. 1, pp. 89-105, 2016.

[35] R. Okada, K. Wakai, M. Naito et al., "Pro-/anti-inflammatory cytokine gene polymorphisms and chronic kidney disease: a cross-sectional study," BMC Nephrology, vol. 13, no. 1, article 2, 2012.

[36] D. V. Barreto, F. C. Barreto, S. Liabeuf et al., "Plasma interleukin6 is independently associated with mortality in both hemodialysis and pre-dialysis patients with chronic kidney disease," Kidney International, vol. 77, no. 6, pp. 550-556, 2010.

[37] P. Stenvinkel, M. Ketteler, R. J. Johnson et al., "IL-10, IL-6, and TNF- $\alpha$ : central factors in the altered cytokine network of uremia - the good, the bad, and the ugly," Kidney International, vol. 67, no. 4, pp. 1216-1233, 2005.

[38] D. J. Oh, H. R. Kim, M. K. Lee, and Y. S. Woo, "Profile of human $\beta$-defensins 1,2 and proinflammatory cytokines (TNF- $\alpha$, IL-6) in patients with chronic kidney disease," Kidney \& Blood Pressure Research, vol. 37, no. 6, pp. 602-610, 2013.

[39] B. Spoto, D. Leonardis, R. M. Parlongo et al., "Plasma cytokines, glomerular filtration rate and adipose tissue cytokines gene expression in chronic kidney disease (CKD) patients," Nutrition, Metabolism and Cardiovascular Diseases, vol. 22, no. 11, pp. 981-988, 2012.

[40] B. Spoto, F. Mattace-Raso, E. Sijbrands et al., "Association of IL-6 and a functional polymorphism in the IL-6 gene with cardiovascular events in patients with CKD," Clinical Journal of the American Society of Nephrology, vol. 10, no. 2, pp. 232-240, 2015.

[41] M. I. Yilmaz, D. Siriopol, M. Saglam et al., "Osteoprotegerin in chronic kidney disease: associations with vascular damage and cardiovascular events," Calcified Tissue International, vol. 99, no. 2, pp. 121-130, 2016.

[42] K. Caidahl, T. Ueland, and P. Aukrust, "Osteoprotegerin: a biomarker with many faces," Arteriosclerosis, Thrombosis, and Vascular Biology, vol. 30, no. 9, pp. 1684-1686, 2010.

[43] P. Demir, F. Erdenen, H. Aral et al., "Serum osteoprotegerin levels related with cardiovascular risk factors in chronic kidney disease," Journal of Clinical Laboratory Analysis, 2016.

[44] M. Morena, A.-M. Dupuy, I. Jaussent et al., "A cut-off value of plasma osteoprotegerin level may predict the presence of coronary artery calcifications in chronic kidney disease patients," Nephrology Dialysis Transplantation, vol. 24, no. 11, pp. 3389$3397,2009$.

[45] M. Mesquita, A. Demulder, F. Wolff et al., "Osteoprotegerin and progression of coronary and aortic calcifications in chronic kidney disease," Transplantation Proceedings, vol. 42, no. 9, pp. 3444-3449, 2010.

[46] J. R. Lewis, W. H. Lim, T. Ueland et al., "Elevated circulating osteoprotegerin and renal dysfunction predict 15 -year cardiovascular and all-cause mortality: a prospective study of elderly women," PLoS ONE, vol. 10, no. 7, Article ID e0134266, 2015.

[47] G. M. London, "Bone-vascular axis in chronic kidney disease: a reality?" Clinical Journal of the American Society of Nephrology, vol. 4, no. 2, pp. 254-257, 2009.

[48] N. K. Lee, H. Sowa, E. Hinoi et al., "Endocrine regulation of energy metabolism by the skeleton," Cell, vol. 130, no. 3, pp. 456469, 2007.

[49] A. N. Kapustin and C. M. Shanahan, "Osteocalcin: a novel vascular metabolic and osteoinductive factor?" Arteriosclerosis, Thrombosis, and Vascular Biology, vol. 31, no. 10, pp. 2169-2171, 2011.

[50] T. Eleftheriadis, C. Kartsios, G. Antoniadi et al., “The impact of chronic inflammation on bone turnover in hemodialysis patients," Renal Failure, vol. 30, no. 4, pp. 431-437, 2008.

[51] D. V. Barreto, A. Lenglet, S. Liabeuf et al., "Prognostic implication of plasma osteopontin levels in patients with chronic kidney disease," Nephron Clinical Practice, vol. 117, no. 4, pp. c363-c372, 2011.

[52] J. M. Lorenzen, H. Neunhöffer, S. David, J. T. Kielstein, H. Haller, and D. Fliser, "Angiotensin II receptor blocker and statins lower elevated levels of osteopontin in essential hypertension-results from the EUTOPIA trial," Atherosclerosis, vol. 209, no. 1, pp. 184-188, 2010. 
[53] P. Evenepoel, B. Meijers, L. Viaene et al., "Fibroblast growth factor-23 in early chronic kidney disease: additional support in favor of a phosphate-centric paradigm for the pathogenesis of secondary hyperparathyroidism," Clinical Journal of the American Society of Nephrology, vol. 5, no. 7, pp. 1268-1276, 2010.

[54] A. Yasin, D. Liu, L. Chau, J. Madrenas, and G. Filler, "Fibroblast growth factor-23 and calcium phosphate product in young chronic kidney disease patients: a cross-sectional study," $B M C$ Nephrology, vol. 14, no. 1, article 39, 2013.

[55] J. J. Scialla, H. Xie, M. Rahman et al., "Fibroblast growth factor23 and cardiovascular events in CKD," Journal of the American Society of Nephrology, vol. 25, no. 2, pp. 349-360, 2014.

[56] J. Chudek, P. Kocełak, A. Owczarek et al., "Fibroblast growth factor 23 (FGF23) and early chronic kidney disease in the elderly," Nephrology Dialysis Transplantation, vol. 29, no. 9, pp. 1757-1763, 2014.

[57] T. Stompór, "Coronary artery calcification in chronic kidney disease: an update," World Journal of Cardiology, vol. 6, no. 4, p. 115, 2014.

[58] M. Wolf, "Update on fibroblast growth factor 23 in chronic kidney disease," Kidney International, vol. 82, no. 7, pp. 737-747, 2012.

[59] V. David, A. Martin, T. Isakova et al., "Inflammation and functional iron deficiency regulate fibroblast growth factor 23 production," Kidney International, vol. 89, no. 1, pp. 135-146, 2016.

[60] L. Desjardins, S. Liabeuf, C. Renard et al., "FGF23 is independently associated with vascular calcification but not bone mineral density in patients at various CKD stages," Osteoporosis International, vol. 23, no. 7, pp. 2017-2025, 2012.

[61] N. X. Chen and S. M. Moe, "Pathophysiology of vascular calcification," Current Osteoporosis Reports, vol. 13, no. 6, pp. 372-380, 2015.

[62] E. R. Smith, M. L. Ford, L. A. Tomlinson et al., "Serum calcification propensity predicts all-cause mortality in predialysis CKD," Journal of the American Society of Nephrology, vol. 25, no. 2, pp. 339-348, 2014. 


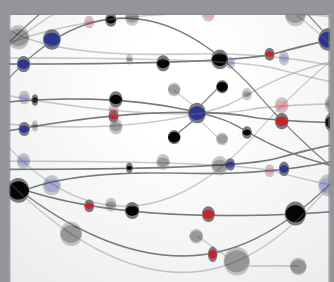

The Scientific World Journal
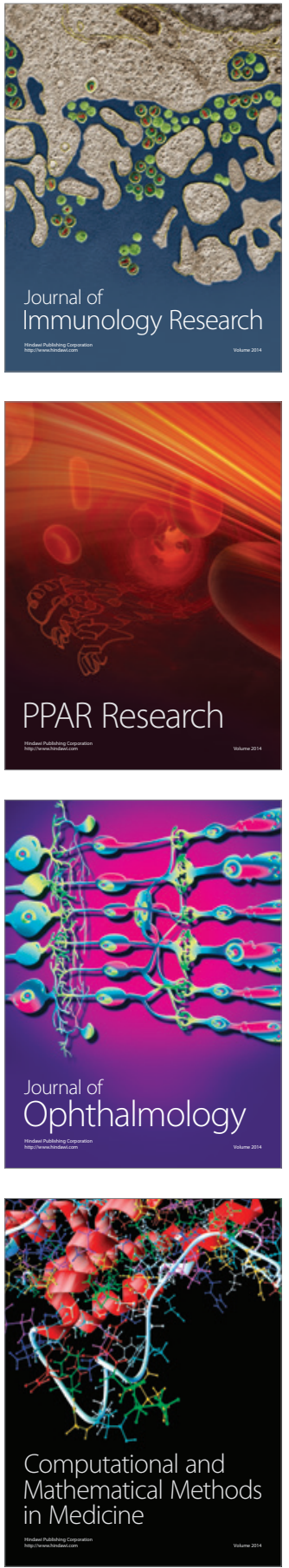

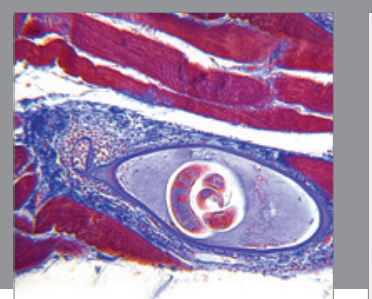

Gastroenterology Research and Practice

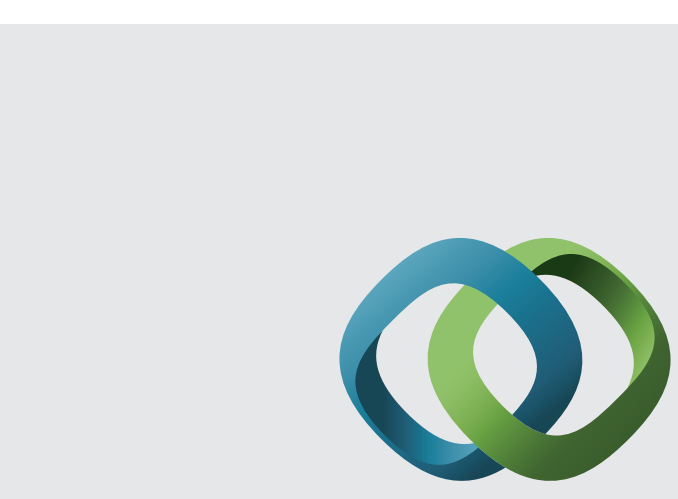

\section{Hindawi}

Submit your manuscripts at

http://www.hindawi.com
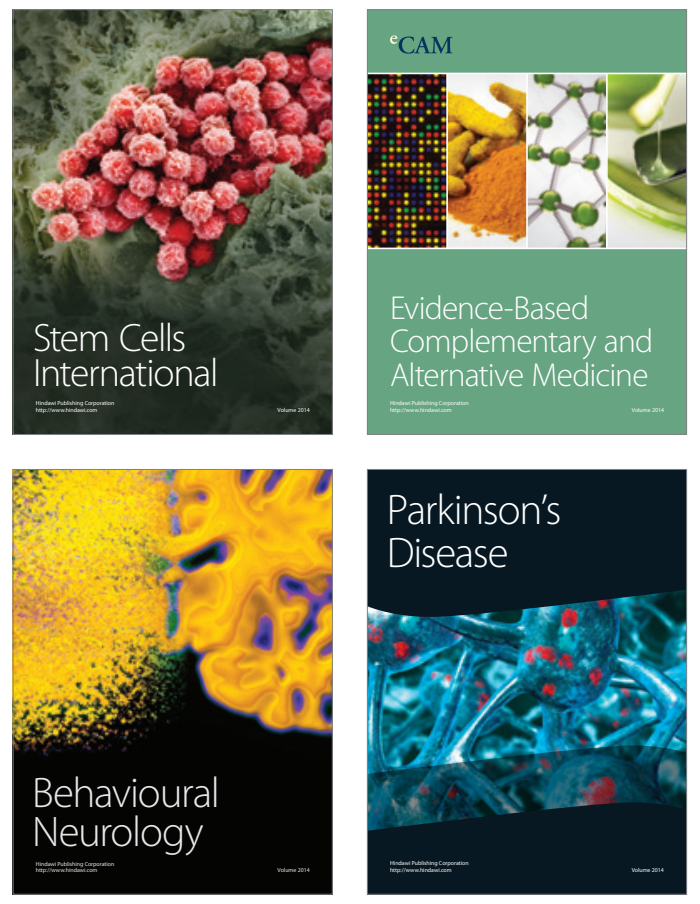
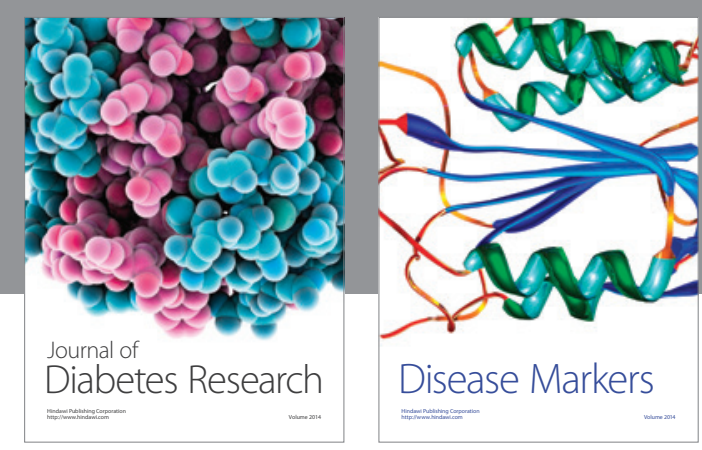

Disease Markers
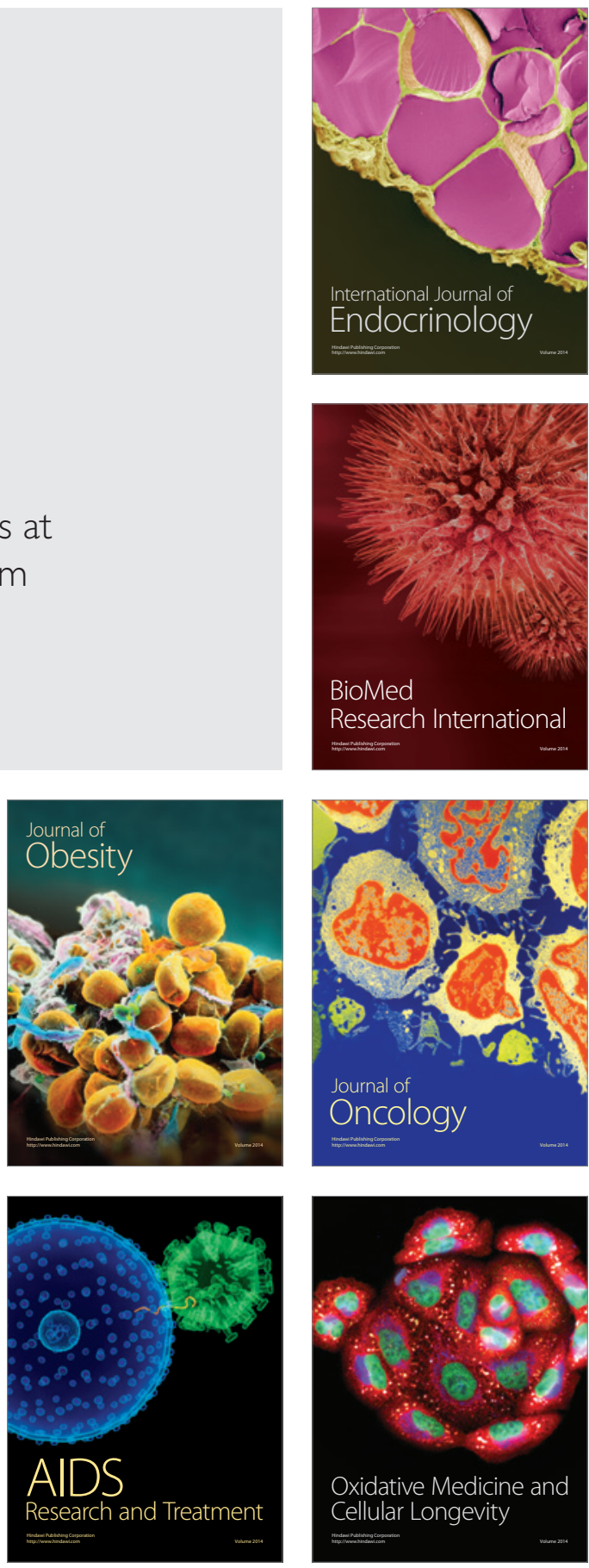